\title{
Optimization of a thick polyvinyl alcohol-acrylamide photopolymer for data storage using a combination of angular and peristrophic holographic multiplexing
}

\author{
Elena Fernández, Celia García, Inmaculada Pascual, Manuel Ortuño, Sergi Gallego, \\ and Augusto Beléndez
}

\begin{abstract}
The capability of polyvinyl alcohol-acrylamide photopolymer materials to obtain angularly multiplexed holographic gratings has been demonstrated [Appl. Phys. B 76, 851 (2003)]. A combination of two multiplexing methods-peristrophic and angular multiplexing-is used to record 60 holograms. An exposure schedule method is used to optimize the capability of the photopolymerizable holographic material and obtain holograms with a higher, more uniform diffraction efficiency. In addition, because of this exposure schedule method, the entire dynamic range $(\mathrm{M} \#)$ of the material will be exploited, obtaining values of approximately $M \# \sim 9$ in layers approximately $800 \mu \mathrm{m}$ thick. (C) 2006 Optical Society of America

OCIS codes: $\quad$ 090.0090, 090.2900, 090.4220.
\end{abstract}

\section{Introduction}

We live in an age of information science and technology in which the use of computers for information processing and storage has become essential. After CD-ROMs, with a capacity to store $0.7 \mathrm{bits} / \mu \mathrm{m}^{2}$, and DVDs, with a capacity to store $4.5 \mathrm{bits} / \mu \mathrm{m}^{2}$, a new field of research has opened up in 3D holographic disks. ${ }^{1-5}$ Investigations are centered on the characterization of new holographic recording materials ${ }^{6}$ and the development of new multiplexing schedules to store information with holographic techniques. The aim of these new techniques is to enable the maximum number of bits $/ \mu \mathrm{m}^{2}$ to be stored. Anderson et al. ${ }^{7}$ has managed to store $150 \mathrm{bits} / \mu \mathrm{m}^{2}$ and Steckman et al. ${ }^{8} 100 \mathrm{bits} / \mu \mathrm{m}^{2}$. For this reason, different methods, such as angular, ${ }^{4,9-11}$ peristrophic, ${ }^{12,13}$ or shift multiplexing $8,14,15$ are being used to store multiple holograms at the same location.

The authors are with the Universidad de Alicante, Apartado 99, E-03080, Alicante, Spain. E. Fernández (elena.fernandez@ua.es), C. García, and I. Pascual are with the Departamento Interuniversitario de Óptica. M. Ortuño, S. Gallego, and A. Beléndez are with the Departamento de Física, Ingeniería de Sistemas y Teoría de la Señal.

Received 7 February 2006; revised 5 May 2006; accepted 12 June 2006; posted 12 June 2006 (Doc. ID 67648).

$0003-6935 / 06 / 297661-06 \$ 15.00 / 0$

(C) 2006 Optical Society of America
In this respect, photopolymers are considered interesting materials for recording holographic memories because they have excellent holographic characteristics, such as a high refractive index modulation of approximately 0.004 to $0.006,{ }^{16,17}$ good light sensitivity, real-time image development, high optical quality, and low cost. In addition, their properties such as energetic sensitivity or spectral sensitivity can be easily changed by modifying the composition.6,9,16

These materials may also have a high dynamic range depending on their thickness. The dynamic range, $M \#$, is a parameter that describes the capacity the material has in a given experimental setup to store holograms.4,6,18 For a material to be used as a holographic memory and have the maximum amount of information stored, the dynamic range must be as high as possible. The higher the dynamic range, the greater the number of holograms that may be stored at the same location with a higher diffraction efficiency.

To fully exploit the diffraction efficiency of the material used, as many holograms as possible need to be stored. In this study two different multiplexing methods are used to increase the number of holograms that may be stored at the same location. The two multiplexing methods used are peristrophic multiplexing (in which the axis is perpendicular to the sample at the intersection of the beams) and angular multiplexing (in which the axis is perpendicular to the plane defined by the two incident laser beams 


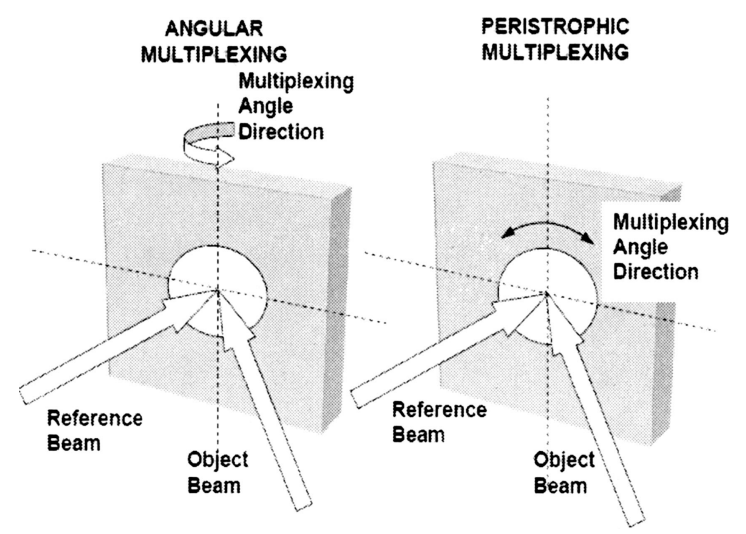

Fig. 1. Schematic of multiplexing methods.

located at the intersection of the beams), shown in Fig. 1. Thus if $n$ holograms may be stored with one of the multiplexing methods and $m$ holograms with the other, combining the two methods makes it possible to store up to $n \times m$ holograms.

The material used to carry out this experiment is a photopolymer based on polyvinyl alcohol- (PVA-) acrylamide..$^{9,16}$ Layers approximately $800 \pm 10 \mu \mathrm{m}$ thick were made, and when 60 holograms were stored, a dynamic range of $M \#=9$ was obtained in them.

In addition to combining two multiplexing methods, the aim of this study is to obtain holograms whose diffraction efficiencies are the same and as high as possible. To achieve this, an iterative method is used to determine the exposure schedule for multiplexing holograms. ${ }^{13}$ To calculate the exposure times necessary for all the holograms to reach the same diffraction efficiency, this method makes use of the dynamic range, the number of holograms stored in the material, the diffraction efficiency of each of the stored holograms, and the exposure energy used to record them.

In Section 2, the method of manufacturing the material is described, together with the holographic setup used to record and read the holograms. In Section 3 , the angular selectivity of the material is measured to determine the angular separation required between the holograms to prevent them from overlapping during storage. The dynamic range of the material is also measured. Subsequently, an exposure schedule is used to calculate the exposure times needed to store holograms with the same, and if possible increased, diffraction efficiency.

\section{Experimental Setup}

The holograms are registered in a photopolymer composed of acrylamide (AA) as the polymerizable monomer, triethanolamine (TEA) as the radical generator, $N, N$ 'methylene-bis-acrylamide (BMA) as the crosslinker, yellowish eosin (YE) as the sensitizer, and a binder of PVA.

The introduction of BMA in the composition improves the energetic sensitivity and the diffraction efficiency of the material and, in addition, gives a greater stability to the stored grating, thereby preventing it
Table 1. Concentrations of the Photopolymer Composition

\begin{tabular}{lc}
\hline & Composition \\
\hline Polyvinyl alcohol & $13.50 \% \mathrm{w} / \mathrm{v}$ \\
Acrylamide & $0.31 \mathrm{M}$ \\
Triethanolamine & $0.12 \mathrm{M}$ \\
Yellowish eosin & $9.10^{-5} \mathrm{M}$ \\
$N, N$ 'methylene-bis-acrylamide & $0.04 \mathrm{M}$ \\
\hline
\end{tabular}

from disappearing with time. Table 1 shows the component concentrations of the photopolymer composition.

A solution of PVA in water forms the matrix, and this is used to prepare the mixture of AA, BMA, and the photopolymerization initiator system composed of TEA and YE. The PVA is supplied by Fluka; the AA, TEA, and BMA by Sigma; and the YE by Panreac. The mixture was made under red light, deposited by gravity in circular polystyrene molds and left in the dark for 6 days to allow the water to evaporate under conditions of temperature, $T$, between $20^{\circ} \mathrm{C}$ and $25^{\circ} \mathrm{C}$, and a relative humidity $(\mathrm{RH})$ of $40 \%-60 \%$.

These conditions of drying time, temperature, and $\mathrm{RH}$ were optimized to obtain the maximum diffraction efficiency in $800 \pm 10 \mu \mathrm{m}$ thick plates. Once dry, they were removed from the mold and placed in a $5.5 \mathrm{~cm} \times 5.5 \mathrm{~cm}$ glass support to be used for recording.

The holographic gratings were recorded by using the output from a diode-pumped frequency-doubled Nd:YAG laser (Coherent Verdi V2), which was split into two beams and then spatially filtered, by using a microscope objective lens and a pinhole, and collimated to yield a plane-wave source of light at $532 \mathrm{~nm}$. The diameters of these beams were $1.5 \mathrm{~cm}$ and the intensity $5 \mathrm{~mW} / \mathrm{cm}^{2}$, with an intensity ratio of $1: 1$. The Gaussian light beams were spatially overlapped at the recording medium intersection at an angle of $17.4^{\circ}$ (measured in air), resulting in an interference grating with a spatial frequency of 1125 lines $/ \mathrm{mm}$. The diffracted intensity was monitored in real time with the $\mathrm{He}-\mathrm{Ne}$ laser positioned at Bragg's angle $\left(\theta^{\prime}=20.8^{\circ}\right)$. To obtain the diffraction efficiency as a function of the angle at reconstruction, the plates were placed on a rotating stage. The diffraction efficiency was calculated as the ratio of the diffracted beam to the incident power (Fig. 2).

\section{Results and Discussion}

First, the energetic sensitivity, the angular selectivity of the material and the dynamic range are measured. To calculate the energetic sensitivity, a single grating is recorded until the diffraction efficiency is saturated. Figure 3(a) shows the diffraction efficiency versus the exposure time. The maximum diffraction efficiency is reached when the material is exposed for approximately $14 \mathrm{~s}$ with an intensity of $5 \mathrm{~mW} / \mathrm{cm}^{2}$. But in this experiment we do not want the diffraction efficiency to reach the maximum; we want a diffraction efficiency of not more than 10\%-15\%. This way, 


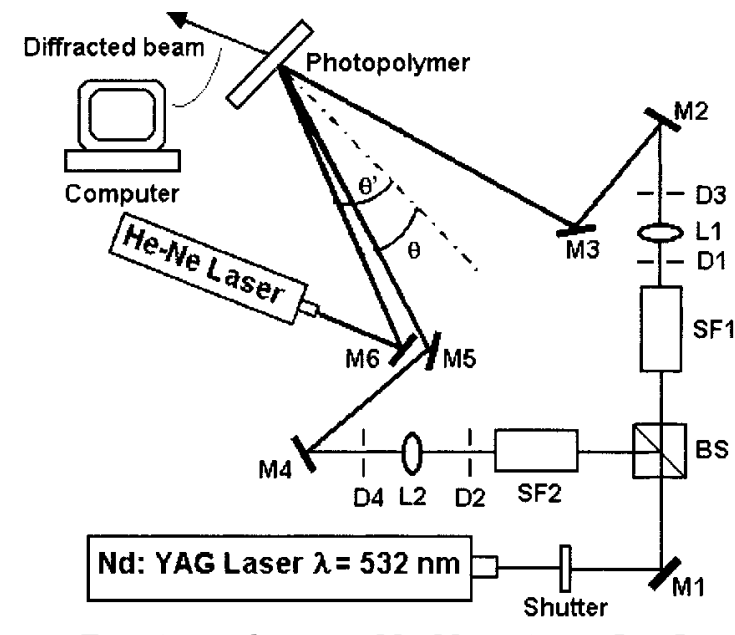

Fig. 2. Experimental setup: M1-M6, mirror; L1, L2, lens; D1-D4, diaphragm; SF1, SF2, microscope objective, lens, and pinhole; BS, beam splitter.

the first holograms do not consume all the dynamic range, and we can record the last ones. With an exposure time of $1 \mathrm{~s}$, a diffraction efficiency of approximately $7 \%$ was reached; for this reason we chose this initial exposure time. The error in the diffraction efficiency measurement in each grating was $0.02 \%$.

To calculate the angular selectivity of the material

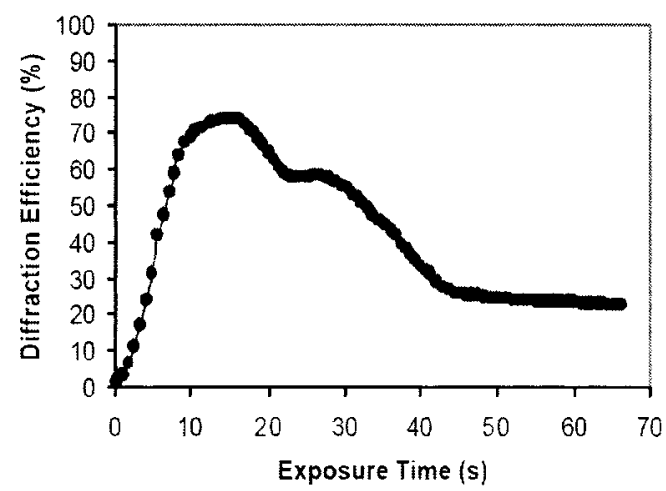

(a)

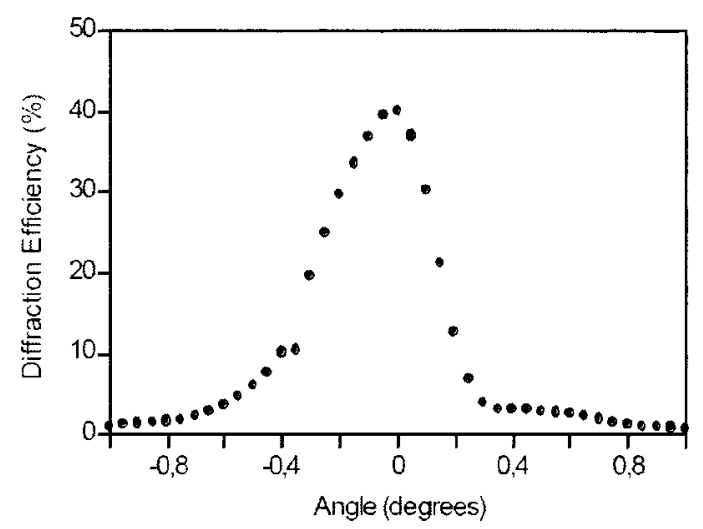

(b)

Fig. 3. (a) Diffraction efficiency versus exposure time. (b) Angular scanning.

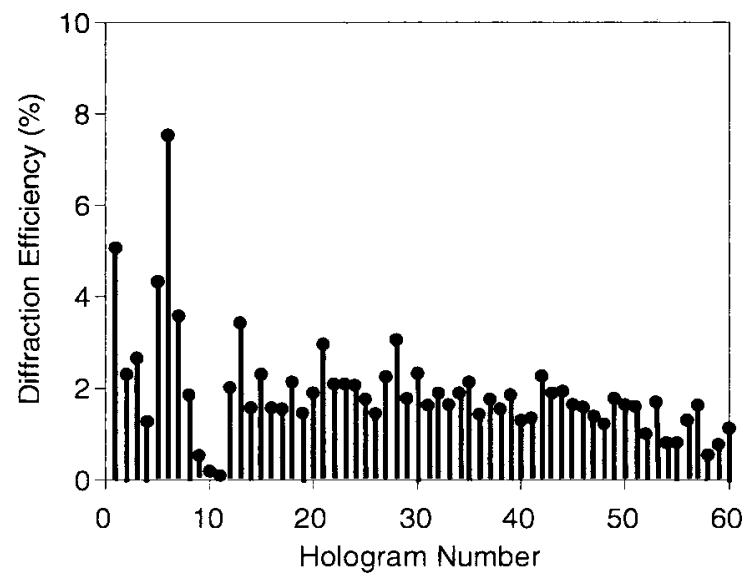

Fig. 4. Diffraction efficiency versus hologram number.

(the angular bandwidth of the main lobe of the stored grating), a hologram was stored in the material and its angular scanning measured [Fig. 3(b)]. From the angular selectivity it was possible to determine the angular separation necessary between the holograms to prevent them from overlapping during storage. The bandwidth of the stored holograms was approximately $0.5^{\circ}$. Therefore they need to be separated by this angular distance to prevent overlapping.

Second, the dynamic range of the material must be measured to determine how many holograms with a specific diffraction efficiency can be stored in the material. To calculate the dynamic range $M \#$, as many holograms as possible are recorded in the material, and the square roots of their diffraction efficiencies are added up. ${ }^{4,6,18}$

In the first experiment, 60 holograms were stored at the same location, and there was interference between two plane waves. A combination of two multiplexing methods-peristrophic and angular-was used to store the holograms. By using angular multiplexing, seven holograms separated by $0.5^{\circ}$ were stored at each peristrophic multiplexing position. Each peristrophic multiplexing position was separated by $10^{\circ}$, and holograms were stored at nine locations. This angular separation for peristrophic multiplexing was chosen randomly, since if we wanted to store a greater number of holograms, they could be stored with a separation of only $1^{\circ}$ and still not overlap.

To store the 60 holograms, the exposure time to be used to record them must be decided upon. As the holograms are stored, the monomer and the dye in the material are consumed. This means that a greater exposure energy is necessary if the last holograms recorded are to have the same diffraction efficiency as the first. Therefore the exposure time used to store the holograms is as follows: $1 \mathrm{~s}$ for the first 20 holograms, and then $0.5 \mathrm{~s}$ is added for every five holograms stored. An exposure time of $1 \mathrm{~s}$ was taken because with this time a diffraction efficiency of approximately $7 \%$ is reached [Fig. 3(a)].

Figure 4 shows the diffraction efficiency obtained when the holograms are recorded with the above exposure times versus the number of holograms stored. 


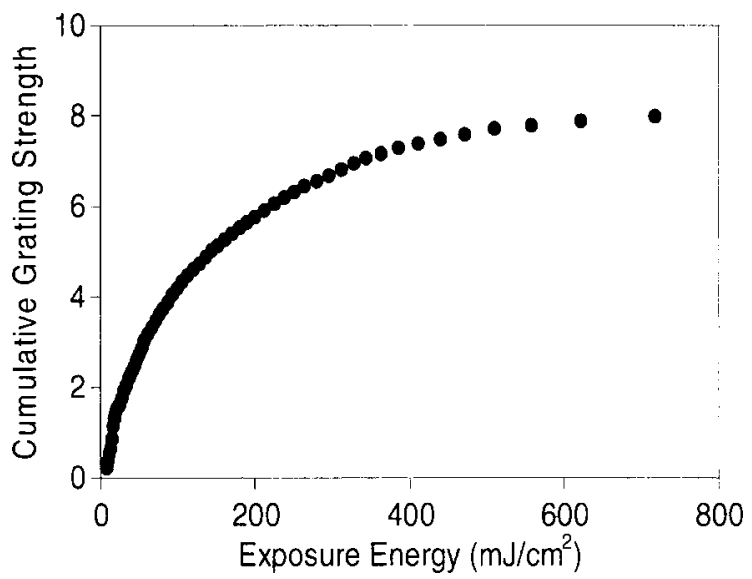

Fig. 5. Cumulative grating strength as a function of exposure energy.

Sixty holograms were stored with a mean diffraction efficiency of $1.8 \%$. As can be seen, the diffraction efficiency of the first five holograms is much higher than the mean. This is because at the beginning the material is very sensitive to the exposure energy used. As the holograms are recorded, the monomer and the dye in the material are consumed and this means that a greater exposure energy is necessary to reach the same diffraction efficiency. Therefore the last holograms stored have a lower diffraction efficiency since, as all the monomer and dye have been consumed, the hologram will not be formed even though the exposure energy is increased.

From the diffraction efficiencies reached in the stored gratings we calculated the cumulative grating strength (the sum of the square roots of the diffraction efficiency $\sum_{i=1}^{N} \eta_{i}^{1 / 2}$, in which $\eta$ is the diffraction efficiency and $N$ is the number of holograms stored so far) and represented it as a function of exposure energy (Fig. 5). As can be seen, when the curve is saturated the dynamic range is $M \#=8$. From the dynamic range and the number of holograms to be stored, it is possible to determine the mean theoretical diffraction efficiency that could be reached for each of the holograms. To calculate the mean diffraction efficiency, the dynamic range $M \#$ was divided by the number of holograms and the result obtained was squared:

$$
\eta_{\text {Avg }}=\left(\frac{M \#}{N}\right)^{2}
$$

Therefore when $M \#=8$ and 60 holograms are stored, a mean theoretical diffraction efficiency of $1.8 \%$ could be achieved, as was obtained experimentally.

To store the 60 holograms with the same diffraction efficiency, an exposure schedule was used to optimize the exposure energy that each hologram should receive to achieve the same diffraction efficiency. ${ }^{13}$ The aim of this exposure schedule is to fully exploit the dynamic range of the material and divide it between all of the holograms. Therefore the exposure schedule is used to calculate the recording times necessary to achieve this uniformity.

This is done as follows: First, the data obtained from Fig. 5 are fitted in the following theoretical equation:

$A=a_{0}+a_{1} E+a_{2} E^{2}+a_{3} E^{3}+a_{4} E^{4}+a_{5} E^{5}+a_{6} E^{6}$,

where $A$ is the cumulative grating strength and $E$ is the exposure energy. Once the coefficients $a_{i}$ have been calculated, the time needed to record the holograms may be calculated from the equation

$$
\begin{aligned}
t_{n}= & A_{\text {sat }} / N I\left[a_{1}+2 a_{2} \sum_{i=1}^{n-1} E_{i}+3 a_{3}\left(\sum_{i=1}^{n-1} E_{i}\right)^{2}+4 a_{4}\left(\sum_{i=1}^{n-1} E_{i}\right)^{3}\right. \\
& \left.+5 a_{5}\left(\sum_{i=1}^{n-1} E_{i}\right)^{4}+6 a_{6}\left(\sum_{i=1}^{n-1} E_{i}\right)^{5}\right]
\end{aligned}
$$

where $A_{\text {sat }}$ is the dynamic range obtained, $N$ is the number of holograms to be stored, $I$ is the recording intensity, and $E_{i}$ is the energy used to record up to the $i$ th hologram.

After applying the exposure schedule, the results obtained are shown in Figs. 6 and 7. Figure 6 shows the diffraction efficiency versus the angle of the seven angular multiplexed holograms for each peristrophic multiplexing position. In Figs. 6(a)-6(i), all of the peaks representing stored holograms have been numbered in the order in which they were recorded. Thus, in Fig. 6(a) the central peak corresponds to the first hologram recorded and is therefore labeled 1 . Then holograms 2-7 were recorded in the order shown in the figure. This was done because in previous experiments better results were obtained when the holograms were stored in this way. Figure 6(a) shows the first seven holograms stored in the peristrophic position $0^{\circ}$. As can be seen, the angular separation of $0.5^{\circ}$ used to store them appears to be insufficient since there is some overlapping, although it is possible to make out the seven maxima corresponding to the seven holograms stored. Figure 6(b) shows holograms 8-14 (peristrophic $10^{\circ}$ ), and in this case only two holograms are seen to overlap, whereas all the others are clearly separated. In Figs. 6(c)-6(i) an angular separation of $0.5^{\circ}$ is seen to be more than sufficient to differentiate one hologram from another. This is because, as more holograms are stored, the optical thickness of the material increases and so the angular selectivity decreases. ${ }^{19}$ Thus, as more holograms are stored, the angular separation between them may be reduced, with the result that there is room for a greater number of holograms. Figure 6(i) shows only four holograms stored because all the monomer and the dye in the material has been consumed. To record more holograms it would be necessary to store them with a lower diffraction efficiency.

We calculated the refractive index modulation by using Kogelnik's coupled-wave theory. To do so, each one of gratings represented in Fig. 6 was fitted by Kogelnik's theory. We obtain a $\Delta n$ value and an optical thickness value from each grating. The total 


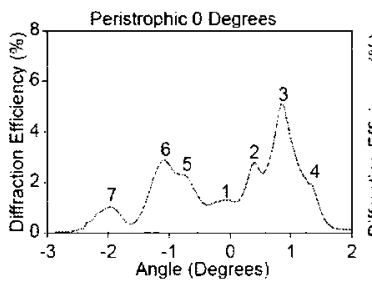

(a)

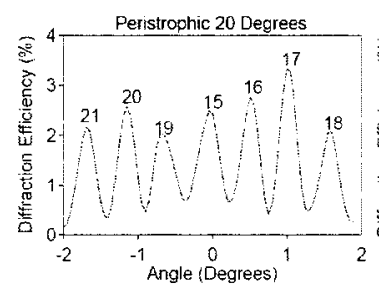

(c)

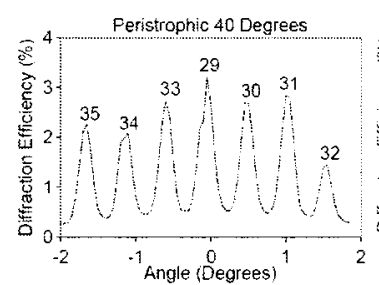

(e)

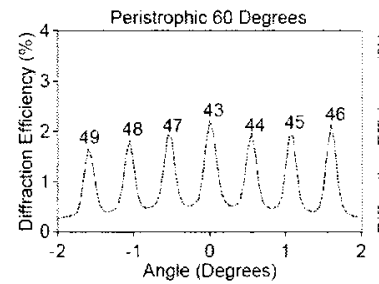

(g)

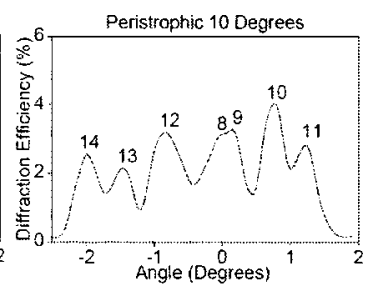

(b)

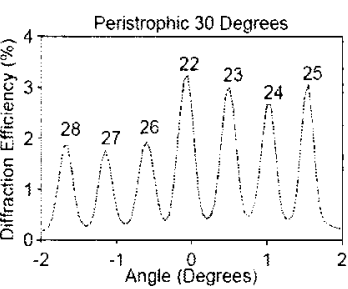

(d)

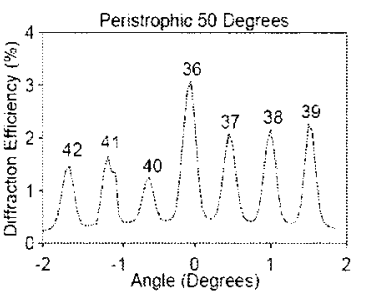

(f)

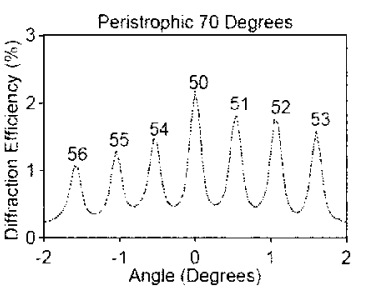

(h)

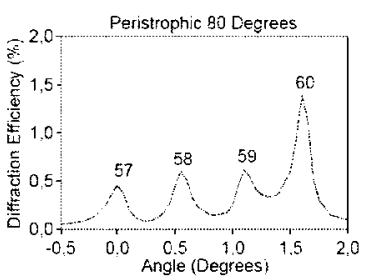

(i)

Fig. 6. Angular scanning of the angularly multiplexed holograms for each position of the peristrophic multiplexing.

refractive index modulation will be the sum of the refractive index modulation of the independent gratings, and the optical thickness will be the median of all of them. The value obtained is $\Delta n=0.0040$ \pm 0.0005 with an optical thickness of $400 \pm 50 \mu \mathrm{m}$. If the optical thickness is smaller than the physical thickness this is due to the attenuated index profile. ${ }^{19}$ In addition, we calculate $\Delta n$ with Eq. (4) (Ref. 4):

$$
\Delta n=\frac{M \# \lambda \cos \left(\theta_{i}\right)}{\pi d}
$$

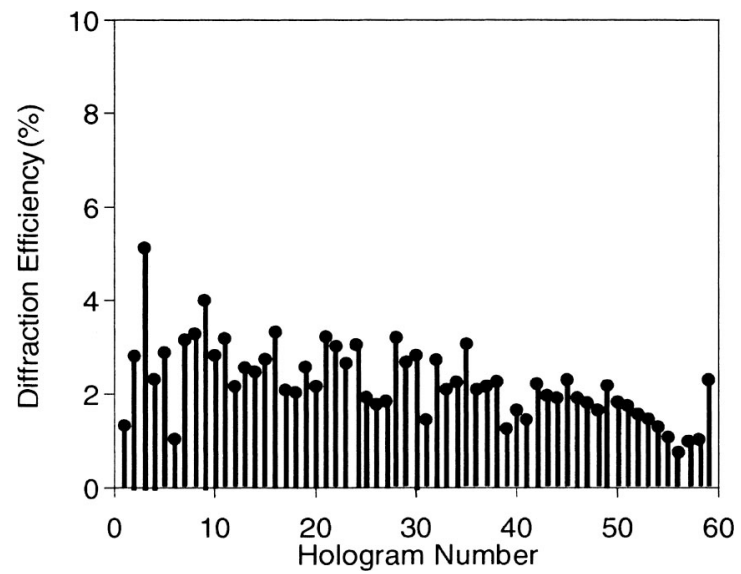

Fig. 7. Diffraction efficiency versus hologram number after applying the exposure schedule.

where $M \#=8.7 \pm 0.1, d=400 \pm 50 \mu \mathrm{m}$ (optial thickness), $\theta_{i}=11.7^{\circ} \pm 0.1^{\circ}(1 / 2$ angle between the writing beams internal to the medium), and $\lambda$ $=532 \mathrm{~nm}$. The value obtained is $\Delta n=0.0036$ \pm 0.0005 . The values of $\Delta n$ obtained with both methods are equal if we consider the error.

Figure 7 shows the diffraction efficiency of the 60 stored holograms versus the hologram number. The first holograms were stored with a diffraction efficiency close to the mean, whereas in Fig. 4 the first hologram had a diffraction efficiency higher than the mean. In this sense the results have improved considerably since, as the maximum diffraction efficiency of the first holograms decreases, more uniform results may be obtained. Moreover, the new exposure times given by the exposure schedule result in a higher mean experimental diffraction efficiency as compared with the results shown in Fig. 4 (2.0\% versus $1.8 \%$ ). Although the increase may seem small, it is actually quite considerable bearing in mind that in other studies the diffraction efficiency did not exceed $0.6 \%$ (Ref. 20 ) or $1.5 \% .10$

By using the data shown in Fig. 7, the dynamic range was once again calculated and found to be $M \#=8.7$. The value of $M \#$ has increased because the dynamic range has been more fully exploited thanks to the new recording times. With this new dynamic range, the mean theoretical diffraction efficiency that can be reached when storing 60 holograms is found to be $2.1 \%$. As in Fig. 4, the last holograms have a slightly lower diffraction efficiency since almost all the monomer and dye have been consumed. If we disregard the last five holograms in the calculation, the mean diffraction efficiency is $2.3 \%$ with a dynamic range of $M \#=8.4$.

With our $800 \mu \mathrm{m}$ thick photopolymer, we obtained a dynamic range of $M \#=8.7$. InPhase Technology 7 has developed a material which has values of $M \#$ between 17 and 50 for $1.5 \mathrm{~mm}$ thick layers. The dependence of $M \#$ on thickness is lineal. ${ }^{6}$ Therefore from this dependence it can be seen that if our material were $1.5 \mathrm{~mm}$ thick, its dynamic range would be $M \#=16$, which is of the same order of magnitude as 
that of the InPhase materials. In addition, Hsu et al. ${ }^{6}$ obtained values of $M \# \sim 2$ for $1 \mathrm{~mm}$ layers. The value obtained in our study is clearly higher.

\section{Conclusion}

The results obtained when 60 holograms are stored in a material based on PVA-acrylamide have been presented. With the composition used to manufacture the material, it has been shown that this material is capable of reaching a dynamic range of $M \#=8.7$, with an angular selectivity of $0.5^{\circ}$. Using these data, two multiplexing methods-angular multiplexing and peristrophic multiplexing-were combined to store 60 holograms, and diffraction efficiencies of $1.8 \%$ were obtained. An exposure schedule was applied to the recorded holograms to calculate the exposure times necessary to obtain uniform diffraction efficiencies. Not only was it possible to obtain uniform diffraction efficiencies, but also the dynamic range was more fully exploited, thereby increasing the mean diffraction efficiency to $2 \%$.

This work was supported by the Ministerio de Educación y Ciencia (Spain) under projects FIS200505881-C02-01, FIS2005-05881-C02-02, and GRE 04-06.

\section{References}

1. A. Pu and D. Psaltis, "High-density recording in photopolymerbased holographic three-dimensional disks," Appl. Opt. 35, 2389-2398 (1996).

2. O. Graydon, "Holographic storage turns blue," Opto. Laser Europe 125, 7 (2005).

3. A. H. Tullo, "Data storage in 3-D," Chem. Eng. News 83, 31-32 (2005).

4. M. Schnoes, B. Ihas, A. Hill, L. Dhar, D. Michaels, S. Setthachayanon, G. Schomberger, and W. L. Wilson, "Holographic storage media for practical systems," in Practical Holography XVII and Holographic Materials IX, T. H. Jeong, ed., Proc SPIE 5005, 29-37 (2003).

5. R. R. McLeod, A. J. Daiber, M. E. McDonald, T. L. Robertson, T. Slagle, S. L. Sochava, and L. Hesselink, "Microholographic optical disk data storage," Appl. Opt. 44, 3197-3207 (2005).

6. K. Y. Hsu, S. H. Lin, and Y.-N. Hsiao, "Experimental characterization of phenanthrenequinode-doped poly(methyl methacrylate) photopolymer for volume holographic storage," Opt. Eng. 42, 1390-1396 (2003).

7. K. Anderson, E. Fotheringham, A. Hill, B. Sissom, and K. Curtis,
"High holographic data storage at $100 \mathrm{Gbits} / \mu \mathrm{in}^{2} "$ (2005), http://www.inphase-technologies.com/technology/whitepapers/ pdfs/high_speed_data_storage.pdf.

8. G. J. Steckman, A. Pu, and D. Psaltis, "Storage density of shift-multiplexed holographic memory," Appl. Opt. 40, 33873394 (2001).

9. M. Ortuño, S. Gallego, C García, C Neipp, A Beléndez, and I. Pascual, "Optimization of a $1 \mathrm{~mm}$ thick PVA/acrylamide recording material to obtain holographic memories: method of preparation and holographic properties," Appl. Phys. B 76, 851-857 (2003).

10. H. Sherif, I. Naydenova, S. Martin, and C. McGinn, "Characterization of an acrylamide-based photopolymer for data storage utilizing holographic angular multiplexing," J. Opt. A, Pure Appl. Opt. 7, 255-260 (2005).

11. I. El Hafidi, R. Grzymala, R. Kiefer, L. Elouad, and P. Meyrueis, "Optical data storage on protein using angular multiplexing," Opt. Laser Technol. 37, 503-508 (2005).

12. K. Curtis, A. Pu, and D. Psaltis, "Method for holographic storage using peristrophic multiplexing," Opt. Lett. 19, 993-994 (1994).

13. A. Pu, K. Curtis, and D. Psaltis, "Exposure schedule for multiplexing holograms in photopolymer films," Opt. Eng. 35, 2824-2828 (1996).

14. G. A. Rakuljic, V. Levya, and A. Yariv, "Optical data storage by using orthogonal wavelength-multiplexed volume holograms," Opt. Lett. 17, 1471-1473 (1992).

15. L. Cao, X. Ma, Q. He, H. Wu, and G. Jin, "Imaging spectral device based on multiple volume holographic gratings," Opt. Eng. 43, 2009-2016 (2004).

16. S. Gallego, M. Ortuño, C. Garcia, C. Neipp, A. Belendez, and I. Pascual, "High-efficiency volume holograms recording on acrylamide and N,N'methilene-bis-acrylamide photopolymer with pulsed laser," J. Mod. Opt. 52, 1575-1584 (2005).

17. S. Gallego, M. Ortuño, C. Neipp, A. Márquez, A. Bélendez, and I. Pascual, "Characterization of polyvinyl alcohol/acrylamide holographic memories with a first-harmonic difusion model," Appl. Opt. 44, 6205-6210 (2005).

18. F. H. Mok, G. W. Burr, and D. Psaltis, "System metric for holographic memory systems," Opt. Lett. 21, 896-898 (1996).

19. S. Gallego, M. Ortuño, C. Neipp, A. Marquez, A. Belendez, I. Pascual, J. V. Kelly, and J. T. Sheridan, "Physical and effective optical thickness of holographic diffraction gratings recorded in photopolymers," Opt. Express 13, 1939-1947 (2005).

20. A. Yan, S. Tao, D. Wang, M. Shi, and F. Wu, "Multiplexing holograms in the photopolymer with equal diffraction efficiency," in Advances in Optical Data Storage, Technology, D. $\mathrm{Xu}, \mathrm{K}$. A. Schouhamer Immink, and K. Shono, eds., Proc SPIE 5643, 109-117 (2005). 